\title{
U-bent plastic optical fiber for lard adulteration sensor in edible oil
}

\author{
Ika Puspitaa,*, Fredy Kurniawan ${ }^{\mathrm{b}}$, Agus Muhamad Hattaa, Sekartedjo Koentjoro ${ }^{\mathrm{a}}$ \\ a Department of Engineering Physics, Faculty of Industrial Technology and System \\ Engineering, Institut Teknologi Sepuluh Nopember, Surabaya, 60111, Indonesia. \\ ${ }^{b}$ Department of Chemistry, Faculty of Science and Data Analytics, Institut Teknologi Sepuluh \\ Nopember, Surabaya, 60111, Indonesia.
}

\begin{abstract}
A plastic optical fiber with the structure of U-bent was utilized as a sensor to detect lard adulterants in olive oil. The macro bending was formed on the plastic optical fiber with a bending radius of $10 \mathrm{~mm}, 12.5 \mathrm{~mm}$, and $15 \mathrm{~mm}$. The output spectra and intensity were measured to detect the existence of lard substance in olive oil. The U-bent plastic optical fiber sensor with a bending radius of $12.5 \mathrm{~mm}$ has the optimum performance to detect the lard adulterant substance in olive oil. It has a sensitivity of 4.6 a.u/\% with $855 \mathrm{~nm}$ LED source and 10.07 a.u/\% with $940 \mathrm{~nm}$ LED source in intensity-based measurement and $0.50 \mathrm{~nm} / \%$ in spectrum-based measurement. The proposed sensor is the potential to give rapid detection on lard adulterant in edible oil.
\end{abstract}

Keywords: Edible oil, Food safety, Halal detection, Optical fiber sensor.

C 2017 Pusat Kajian Halal ITS. All rights reserved.

\section{Introduction}

Optical fiber is immensely applied as chemical/biosensors [1-6]. Its ease of fabrication and implementation, lightweight, high sensitivity, low cost, and immunity to electromagnetic interference are the main advantages of optical fiber as a sensor in comparison to conventional sensors [7]. As a chemical/biosensor, an optical fiber acts as both a sensing element and a collecting element [8]. It provides efficiency to a detection process. The presence of analyte on the optical fiber surrounding changes the properties of optical fiber and enabling detection. By utilizing its advantages, an optical fiber sensor was designed to detect lard adulteration in olive oil.

As reported by food and drug associations of some countries for the last three decades, some edible oil products were found adulterated by cheap substances including lard or other animal fats, and mislabeled [9-12]. The presence of the lard in edible oil products gives concern to Muslim and Jews consumers in choosing food products. Since it is prohibited for Muslims and Jews to consume products that contain pig or pig derivatives [13].

\footnotetext{
* Corresponding author. Tel: 085259836015; Fax: -.

Email address: ika.tf10@gmail.com
} 
In our previous study, some structures of optical fiber sensors were applied to detect lard adulteration in edible oils and they showed great performance [14-17]. However, it is important to explore the potency of other structures of optical fiber sensors to obtain better performance of the optical fiber sensor. In this paper, a U-bent structure will be formed in a plastic optical fiber with various bending radius. The $U$-bent structure enables evanescence waves from the optical fiber to interact with the surrounding medium. Hence, it allows the detection.

\section{Materials and methods}

\subsection{Sample Preparation}

The pig fat provided in a local market was heated up and melted. The liquid phase of pig fat is then called lard. Then lard was filtered to remove the residual chunks of fat which cannot melt and another unwanted particle. The purified lard was added to olive oil which was purchased from the local store with a concentration of $0-5 \%(\mathrm{v} / \mathrm{v})$. The lard and olive oil mixed by using ultrasonic bathing for 30 minutes to get the homogenous mixture.

\subsection{Experiments}

The U-bent optical fiber sensor is fabricated by forming a macro bending to a multimode polymethyl methacrylate (PMMA) optical fiber with a certain bending radius. A plastic optical fiber from Super Eska TM, SH-4001 has core diameter and cladding thickness of $980 \mu \mathrm{m}$ and $20 \mu \mathrm{m}$, respectively. The jacket of the plastic optical fiber was removed by using an optical fiber jacket cleaver. Then its coating was removed by using acetone with a concentration of $99 \%$. The uncoated part of the plastic optical fiber acts as the sensing element as shown in Fig.1(a). The plastic optical fiber has a refractive index of the core of 1.49 and a numerical aperture of 0.50 . The bending radius was varied $(10,12.5,15 \mathrm{~mm})$ to evaluate the performance of the sensor. The experimental setups are presented in Fig. 1(b)-(c). The U-bent plastic optical fiber was immersed in the sample for both measurements. The spectrum-based measurement as shown in Fig.1(b) will measure the output spectrum of the proposed optical fiber sensor at the wavelength range of $350-1000 \mathrm{~nm}$ as the change of lard concentration in olive oil. The output spectrum was measured for 5 repetitions using Ocean Optics Spectrometer. While, for intensity-based measurement, the infrared LED with the wavelength of $855 \mathrm{~nm}$ and $940 \mathrm{~nm}$ was used as the light source as illustrated in Fig.1(c). The LED sources were driven by Arduino to give stable voltage. The output intensity of the U-bent plastic optical fiber sensor was recorded for 100 seconds using Thorlabs optical power meter. All the experiments were conducted at a room temperature of $20^{\circ} \mathrm{C}$. Since the room temperature affects the properties of the samples and the sensor performance [18]. 


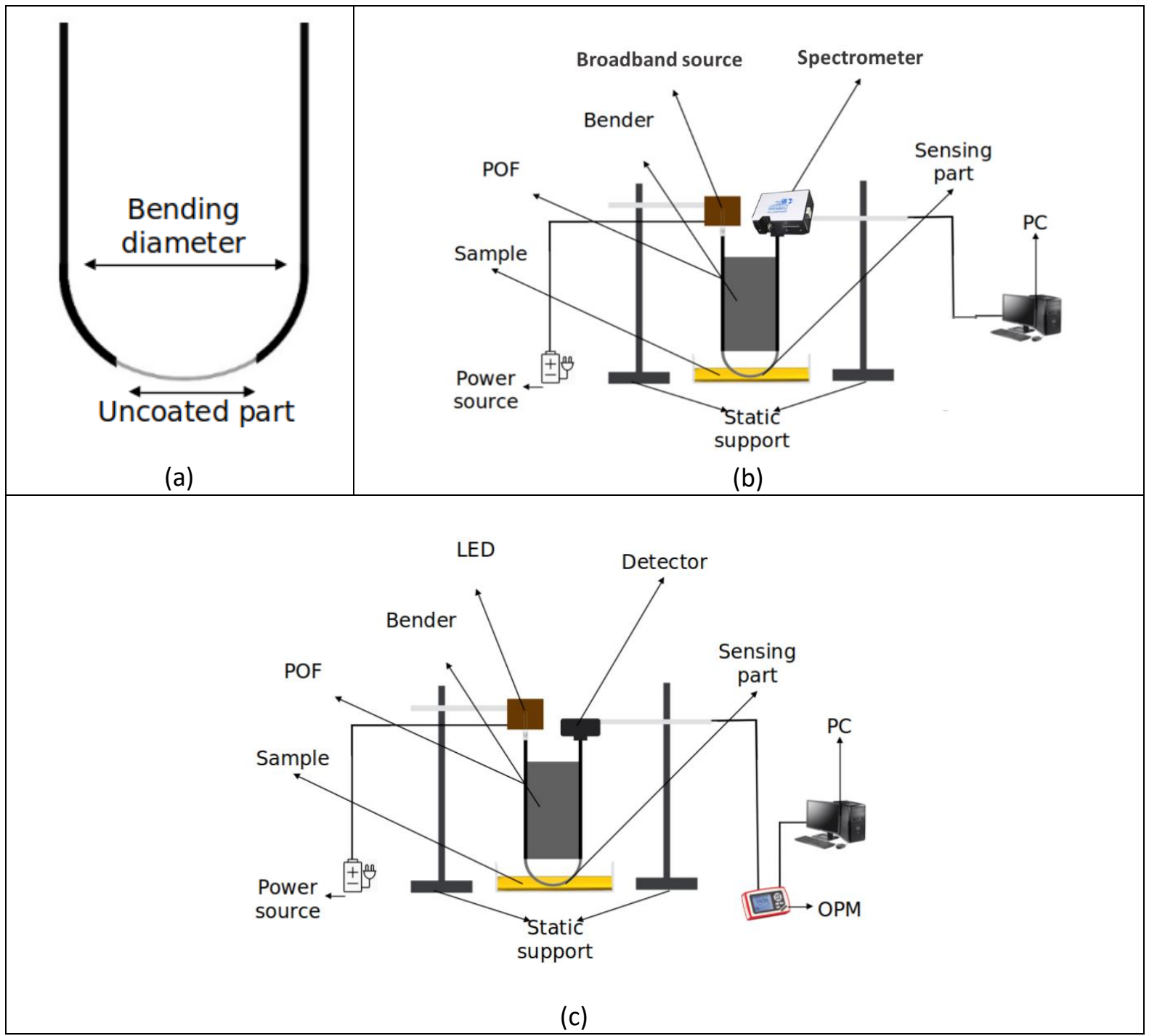

Fig. 1 (a) Schematic diagram of U-bent optical fiber (b) Experimental setup for spectrumbased measurement (c) Experimental setup for intensity-based measurement.

\section{Results and discussion}

The measured output spectra of U-bent plastic optical fiber sensor with various bending radius are presented in Fig. 2 . One can see, the U-bent plastic optical fiber transmitted light which has wavelength peaks at $855 \mathrm{~nm}$ and $940 \mathrm{~nm}$. The change in bending radius of plastic optical fiber does not cancel any wavelength of the light source. However, it has a different response toward a variation of lard concentration in olive oil.

The change of lard concentration in olive oil shifted the peak wavelength of the light source which is transmitted in the U-bent plastic optical fiber at all variations of bending radius. In Fig. 2(a), as the lard concentration increased, the measured spectra the peak wavelength of $855 \mathrm{~nm}$ experience blue-shifting with the sensitivity of $0.5 \mathrm{~nm}$ while the peak wavelength of $940 \mathrm{~nm}$ does not shift. On the contrary, a U-bent plastic optical fiber sensor with a bending radius of $15 \mathrm{~mm}$. It has a blue-shifting response at the wavelength peak of $940 \mathrm{~nm}$ with a sensitivity of $0.5 \mathrm{~nm}$, yet it does not shift the wavelength peak at $855 \mathrm{~nm}$ as depicted in Fig. 2(c). 


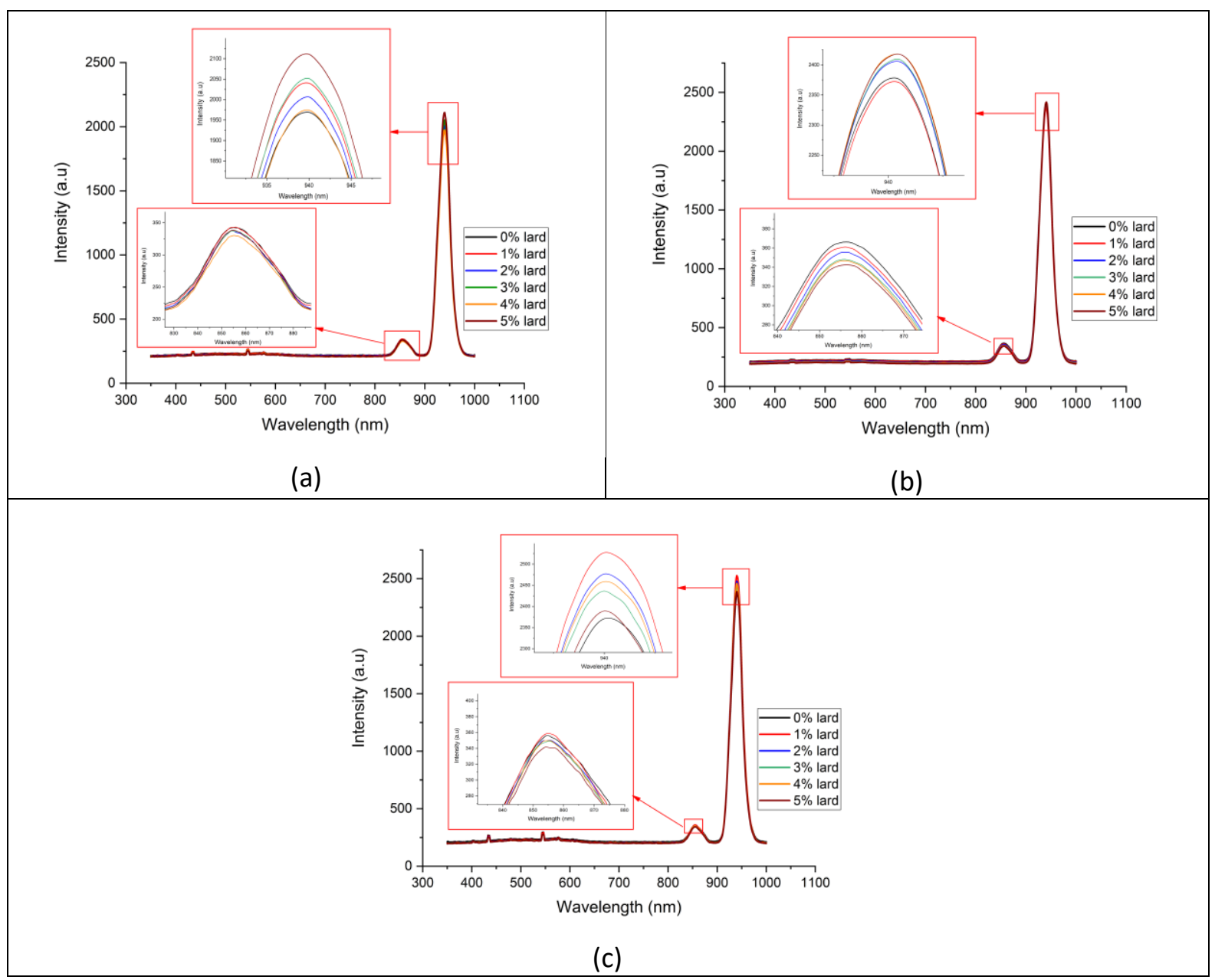

Fig. 2 Measured output spectra of U-bent optical fiber with the radius of (a) $10 \mathrm{~mm}$ (b) 12.5 $\mathrm{mm}$ (c) $15 \mathrm{~mm}$.

A different response showed by U-bent plastic optical fiber with a bending radius of $12.5 \mathrm{~mm}$. The wavelength shifting occurred in both peaks. It shifted to the shorter wavelength at the peak of $855 \mathrm{~nm}$ with the sensitivity of $0.5 \mathrm{~nm}$ while at the peak of $940 \mathrm{~nm}$ is shifted to a longer wavelength with the sensitivity of $0.5 \mathrm{~nm}$.

The addition of lard substance in olive oil changed its refractive index. The change of medium refractive index surrounding the U-bent plastic optical fiber leads to the change of effective refractive index of the light propagation inside the optical fiber. It yields the shifting of the transmitted light wavelength which the results agree with the previous studies [19-21].

The intensity-based measurement was carried out as well to provide a compact-designed measurement. As shown in spectrum-based measurement that the wavelength of $855 \mathrm{~nm}$ and $940 \mathrm{~nm}$ gave a point of interest to be explored in an intensity-based measurement. Hence, the wavelength of $855 \mathrm{~nm}$ and $940 \mathrm{~nm}$ were utilized to investigate the sensor performance. One can see in Fig. 3(a), with an $855 \mathrm{~nm}$ LED, the measured intensity of U-bent plastic optical fiber decrease as the lard concentration in olive oil increased. While, with a 940 $\mathrm{nm}$ LED, it increased as the lard concentration increased. U-bent plastic optical fiber with a 
bending radius of $12.5 \mathrm{~mm}$ has the highest sensitivity in both wavelengths. It has a sensitivity of 4.6 a.u/\% and 10.07 a.u/\% at the wavelength of $855 \mathrm{~nm}$ and $940 \mathrm{~nm}$, respectively.

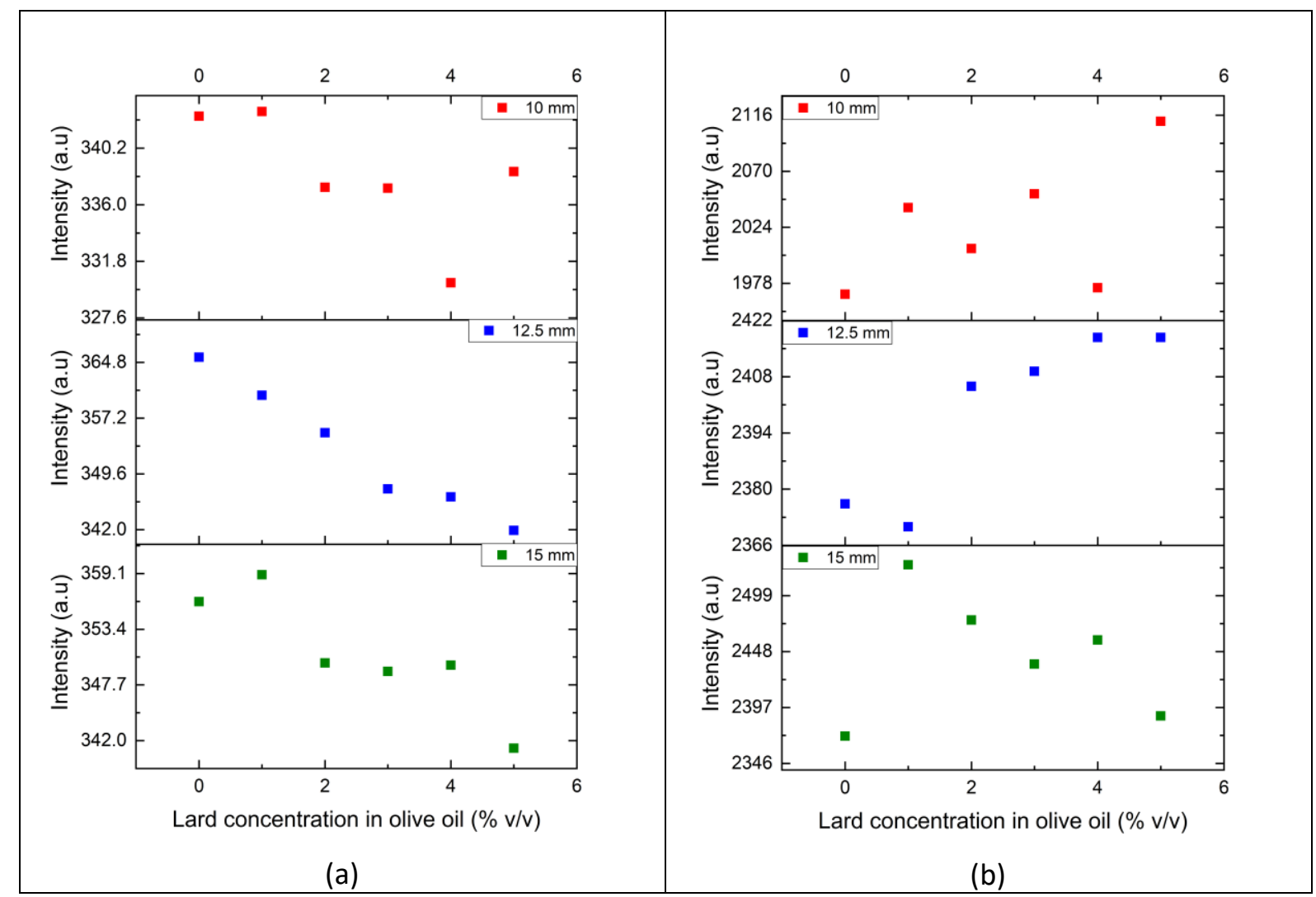

Fig. 3 Measured output intensity of U-bent optical fiber with various radius at a wavelength

$$
\text { of (a) } 855 \mathrm{~nm} \text { (b) } 940 \mathrm{~nm} \text {. }
$$

The U-bent plastic optical fiber sensor exploits the evanescence phenomenon caused by the macro bending on the optical fiber. The variation of the analyte refractive index varied the evanescent modes and their penetration depth to the analyte which acts as the cladding on the sensing element. The simultaneous variation in bending radius and the analyte refractive index caused the different responses of the U-bent plastic optical fiber sensor.

The results show the potency of the U-bent plastic optical fiber sensor to detect lard adulteration in olive oil. The proposed optical fiber sensor provides high sensitivity, low cost, and ease of fabrication method.

\section{Conclusion}

U-bent plastic optical fiber sensor for lard adulteration in olive oil was investigated experimentally. The measured output spectrum and intensity were used to evaluate the performance of the proposed sensor. From all the experiments conducted, the result showed that the U-bent plastic optical fiber sensor for lard adulteration in olive oil has great sensitivity and linearity with a bending radius of $12.5 \mathrm{~mm}$. It has a sensitivity of 4.6 a.u/\% by using an $855 \mathrm{~nm}$ LED source and 10.07 a.u/\% by using a $940 \mathrm{~nm}$ LED source. It can be used as the novel rapid and compact alternative to do oil product authentication. 


\section{Acknowledgments}

This study was funded by Kemenristek DIKTI in the fundamental research scheme 2019 under a contract number of 1202/PKS/ITS/2020.

\section{References}

[1] B.T. Wang and Q. Wang, "An interferometric optical fiber biosensor with high sensitivity for IgG/anti-IgG immunosensing," Opt. Commun., vol. 426, pp. 388-394, 2018, doi: 10.1016/j.optcom.2018.05.058.

[2] N. Cennamo, L. Zeni, E. Ricca, R. Isticato, V.M. Marzullo, A. Capo, M. Staiano, S. D’Auria, and A. Varriale, "Detection of naphthalene in sea-water by a label-free plasmonic optical fiber biosensor," Talanta, vol. 194, pp. 289-297, 2019, doi: 10.1016/j.talanta.2018.10.051.

[3] A. George, M.S. Amrutha, P. Srivastava, S. Sunil, V.V.R. Sai, and R. Srinivasan, "Development of a U-bent plastic optical fiber biosensor with plasmonic labels for the detection of chikungunya non-structural protein 3," Analyst, vol. 146, pp. 244-252, 2021, doi: 10.1039/D0AN01603A.

[4] Y.N. Zhang, T. Zhou, B. Han, A. Zhang, and Y. Zhao, "Optical bio-chemical sensors based on whispering gallery mode resonators," Nanoscale, vol. 10, no. 29, pp. 13832-13856, 2018, doi: 10.1039/C8NR03709D.

[5] K.J. Kim, P. Lu, J.T. Culp, and P.R. Ohodnicki, "Metal-organic framework thin film coated optical fiber sensors: a novel waveguide-based chemical sensing platform," ACS Sens., vol. 3, no. 2, pp. 386-394, 2018, doi: 10.1021/acssensors.7b00808.

[6] H.E. Joe, H. Yun, S.H. Jo, M.B.G. Jun, and B.K. Min, "A review on optical fiber sensors for environmental monitoring," Int. J. of Precis. Eng. and Manuf.-Green Tech., vol. 5, no. 1, pp. 173-191, 2018, doi: 10.1007/s40684-018-0017-6.

[7] M. Ahmad, K.P. Chang, T.A. King, and L.L. Hench, "A compact fibre-based fluorescence sensor," Sens. Actuators A Phys., vol.119, no. 1, pp. 84-89, 2005, doi: 10.1016/j.sna.2004.09.007.

[8] K.N. Sharma and B.D. Gupta, "Fabrication and characterization of pH sensor based on side polished single mode optical fiber," Opt. Commun., vol. 216, no. 4-6, pp. 299-303, 2003, doi: 10.1016/S0030-4018(02)02343-x.

[9] N.H. Jenkins, Virgin Territory-Exploring the World of Olive Oil. New York, USA: Houghton Mifflin Harcourt, 2015.

[10] M. Tejada. "Greek police make arrests in olive oil fraud ring." Olive Oil Times. https://www.oliveoiltimes.com/el/world/greek-police-investigate-olive-oil-stolen-atgunpoint/36395 (accessed May 30, 2017).

[11] N. Blechman. "Extra virgin suicide-the adulteration of Italian olive oil." The New York Times. http://www.nytimes.com/interactive/2014/01/24/opnion/food-chains-extravirgin-suicide.html (accessed May 24, 2017).

[12] G.J. Peng, M.H. Chang, M. Fang, C.D. Liao, C.F. Tsai, S.H. Tseng, Y.M. Kao, H.K. Chou, and H.F. Cheng, "Incidents of major food adulteration in Taiwan between 2011 and 2015," Food Control, vol. 72, pp. 145-152, 2017.

[13] P. Ulca, H. Balta, İ. Çağın, and H.Z. Senyuva, "Meat species identification and Halal authentication using PCR analysis of raw and cooked traditional Turkish foods," Meat Sci., vol. 94, no. 3, pp. 280-284, 2013. 
[14] I. Puspita, F.I. Husada, Sekartedjo, and A.M. Hatta, "Detection of lard adulteration in olive oil by using tapered Plastic Optical Fiber (POF) spectroscopy," in Proc. SPIE-International Society for Optics and Photonics, vol. 11044, 2019.

[15] I. Puspita, D.P.M. Banurea, N. Irawati, Sekartedjo, A.M. Hatta, and F. Kurniawan, "Taper parameters effect on tapered POF for lard adulteration in olive oil detection," Optoelectron. Adv. Mater. Rapid Commun., vol. 14, no. 5-6, pp. 250-255, 2020.

[16] F.I. Husada, I. Puspita, A.M. Hatta, and Sekartedjo, "Detection of lard adulteration in palm oil using U-shaped plastic optical fiber," in Proc. SPIE- International Society for Optics and Photonics, vol. 11044, 2019.

[17] I. Puspita, D. P. M. Banurea, R.N. Hidayati, N. Irawati, M. Marinah, Sekartedjo, A.M. Hatta, and F. Kurniawan, "Detection of lard adulteration in olive oil by using silica optical fiber." 2018 3rd International Seminar on Sensors, Instrumentation, Measurement and Metrology (ISSIMM), IEEE, 2018.

[18] L. Coelho, D. Viegas, J.L. Santos, and J.M.M.M. de Almeida, "Detection of extra virgin olive oil thermal deterioration using a long period fibre grating sensor coated with titanium dioxide," Food Bioproc. Tech., vol. 8, no. 6, pp. 1211-1217, 2015.

[19] C.R. Biazoli, S. Silva, M.A. Franco, O. Frazão, and C.M. Cordeiro, "Multimode interference tapered fiber refractive index sensors," Appl. Opt., vol. 51, no. 24, pp. 5941-5945, 2012.

[20] L.L. Xue and L. Yang, "Sensitivity enhancement of RI sensor based on SMS fiber structure with high refractive index overlay," J. Light. Technol., vol. 10, pp. 1463-1469, 2012.

[21] Q. Wu, Y. Semenova, P. Wang, and G. Farrell, "High sensitivity SMS fiber structure based refractometer-analysis and experiment," Opt. Express, vol. 19, no. 9, pp. 7937-7944, 2011. 